\title{
Modelo de persona, familia y comunidad en salud (MINSA) en el marco del Plan Nacional de Desarrollo Humano (PNDH) de Nicaragua
}

\author{
William Alfonso Blandon Ortiz ${ }^{1}$ \\ Holman Ariel Zamora Herrera ${ }^{2}$ \\ Gerald Marcelino Zelaya Hernández ${ }^{3}$ \\ Augusto Alexander Centeno Garmendia ${ }^{4}$ \\ Beverly Castillo Herrera ${ }^{5}$
}

\section{RESUMEN}

El pensamiento del desarrollo con equidad ha sido dinámico, siguiendo los inmensos cambios de la realidad económica y social. El desarrollo sostenible ha emergido como el principio para el desarrollo mundial de manera equilibrada con el medio ambiente. El Desarrollo Humano es un paradigma en el que las personas puedan desarrollar su máximo potencial, de acuerdo con sus necesidades e intereses que estos demanden. La participación ciudadana es un proceso y prácticas sociales de diversa índole, involucrando los espacios de procesos participativos del quehacer político definiéndose como una cultura, para el fortalecimiento de los actuales sistemas políticos. El nuevo modelo (PNDH) tiene como objetivo las transformaciones estructurales y la superación de la pobreza en Nicaragua implementando estrategias donde se involucre toda la comunidad con programas y proyectos que permitan el desarrollo económico, social de las familias. El Modelo de Salud Familiar y Comunitario está basado en la estrategia de atención primaria en salud, entendida como la asistencia sanitaria basada en el alcance de todas las personas y familias para la mejora de salud de la comunidad. Satisfaciendo las necesidades y el ejercicio de los derechos humanos y contribuyendo a la mejora de su calidad de vida durante el ciclo vital.

Palabras Claves: Desarrollo, participación ciudadana, Nicaragua, salud, familias

Recibido: 17 de noviembre de 2016

Aceptado: 16 de diciembre de 2016

1 UNAN-Managua/FAREM-Estelí. Correo electrónico: william.bland12@gmail.com 2 UNAN-Managua/FAREM-Estelí. Correo electrónico: zamora0610holman@gmail.com 3 UNAN-Managua/FAREM-Estelí. Correo electrónico: heraldzelya96@gmail.com 4 UNAN-Managua/FAREM-Estelí. Correo electrónico: alexandercentenogarmendia@gmail.com

5 Doctora en Ciencias Sociales de la Universidad de Zulia. Docente titular de UNAN-Managua/FAREM-Esteli. Correo electrónico: beverly.castillo@yahoo.com 


\title{
Model of person, family and community in health (MINSA) in the framework of the National Human Development Plan (PNDH) of Nicaragua
}

\begin{abstract}
The thought of the development with equality has been dynamic, following the huge changes of the economic and social reality. Sustainable development has come as the beginning to the world development in a balanced way with the environment. The human development their full potential according to their needs and interests that people demand. The society's participation is a process and different sociable practices, involving the spaces of active processes in the politics actions where it is meant as a culture for strengthening of the current politics systems. The new model (PNDH) has a goal the structural changes and decreasing of Nicaragua's poverty, putting in practice strategies where all communities which work with programs and projects about economic development be involved. The family and community health model is based on primary assisting strategies in health, understood as the sanitary attention based on the best acquisition of families and people in order to get a better health in the community. Satisfying the needs and the actions of the Human rights and forming part of the construction of it quality of life in the vital cycle.
\end{abstract}

Keywords: Development, society participation, Nicaragua, health, families. 


\section{INTRODUCCIÓN}

La forma principal de crecimiento de los países Latinoamericanos llego hasta los años treinta y se basaba en la expansión de las exportaciones mediante el aprovechamiento de las ventajas que ofrecían determinados recursos naturales que era posible colocar en el mercado internacional gracias a la incorporación de dosis masivas de capital y modernas tecnologías, por parte de las grandes potencias. Ante la ruptura del dinámico sistema de comercio exterior que prevalecía antes de la crisis de 1930. Ante las crecientes necesidades de una población cuya tasa de incremento demográfico aumentaba, se hizo evidente la exigencia de desarrollar la producción interna para abastecerla de los bienes y servicios que un sector externo muy poco dinámico (Martner \& Mattar, 2012). Por ello, se fue reafirmando la necesidad de dar mayor coherencia y orientación a estos procesos, y comenzó a abrirse paso rápidamente la idea de recurrir a técnicas de formulación de la política económica que permitieran fijar con suficiente detalle metas de crecimiento que fueran compatibles entre sí y con los recursos con que se contaba.

Surgióentonceslapreocupaciónrelativaalacomplejidad de la tarea de formular un plan de desarrollo personal, con que se enfrentaría el intento de llevar aquella idea a la práctica. Era necesario, por una parte, elaborar una técnica de diagnóstico y proyecciones del desarrollo de los países y aplicarla a casos concretos, resolviendo los problemas consiguientes de disponibilidad de información; $y$, por otra, debían entrenarse numerosos técnicos que pudieran llevar a cabo las tareas inherentes a esas aplicaciones. Se trataba ya no sólo de formular planes de desarrollo con metas globales y sectoriales, sino también de elaborar los elementos y montar los mecanismos que vinculen estos planes en forma más directa con las decisiones económicas y sociales (Martner \& Mattar, 2012). Por ello se organizaron en varios países oficinas centrales de planificación, se elaboraron planes de inversiones públicas y presupuestos por programa y se crearon en algunos casos otros mecanismos parciales. El pensamiento del desarrollo, ha sido dinámico, siguiendo los inmensos cambios de la realidad económica, social y política, regional y mundial. Desde sus primeros años desarrolló un método analítico propio y un énfasis temático que, con algunas variantes, se ha mantenido hasta nuestros días.

\section{Tipos de desarrollo en Latinoamérica.}

Transcurrido medio siglo desde su fundación, la Comisión Económica para América Latina y el Caribe (CEPAL) ha hecho aportes relevantes para el desarrollo regional y sus teorías y visiones han sido escuchadas en muchos lugares del mundo. La CEPAL ya es hoy referencia obligada para quienes estudian la historia económica de la región en el último tiempo. La Comisión se Desarrolló como una escuela de pensamiento especializada en el examen de las tendencias económicas y sociales de mediano y largo plazo de los países latinoamericanos y caribeños. (CEPAL, 2013)

En ella se destaca en primer lugar la búsqueda de una visión integral del desarrollo, de carácter interdisciplinario. Segundo, un juego dialéctico entre ideas y realidad, entre pensamiento y acción, que implica una constante transformación de las primeras en función de los cambios en el entorno, en clara interacción con los gobiernos de la región. Tercero, la búsqueda de una identidad regional, el estudio de la realidad en función de los intereses regionales y el cuestionamiento de cualquier esquema conceptual en función de su validez para comprender nuestras realidades y operar sobre ellas (Sanchez, 2009)

Esas posiciones indican que la CEPAL insiste en sus ideas de regionalismo abierto, apuntando a un modelo regional de cooperación y liberalización comercial al estilo de la organización de cooperación de Asia y Pacífico. No entiende que esas ideas, y ese tipo de 
vinculación, no solo no han funcionado adecuadamente en nuestro continente, sino que la crisis actual las ha puesto en jaque. Al contrario de esa perspectiva, un problema clave de la inserción internacional latinoamericana es superar su vinculación subordinada a la globalización y romper con la persistente privatización de sus economías, donde continuamos siendo exportadores de materias primas. Es así que las distintas comisiones deberían apoyar otra integración regional, como paso indispensable para poder ensayar desvinculaciones selectivas con la globalización y recuperar la autonomía en diseñar estrategias propias de desarrollo que no dependen solamente de los mercados exportadores o de los inversores extranjeros que sean más desarrollo social, se deben elaborar informes de que tipo de desarrollo queremos como sociedad latinoamericana. (Gudynas, 2009).

El propósito de este artículo es dar a conocer los diferentes tipos de desarrollo implantados en América Latina y sus repercusiones, el grado de efectividad y fracaso que se ha obtenido. Y de cómo se produce la preocupación de crear nuevas políticas sociales y económicas sostenible donde se vea un mayor grado de participación ciudadana como el principal eje de cambio. Es por eso que el Gobierno de Reconciliación y Unidad Nacional (GRUN) en su Plan Nacional de Desarrollo Humano (PNDH) considera a las familias como las principales protagonistas de este proyecto revolucionario. Esto, se ve en reflejado en el nuevo modelo de salud que el GRUN está impulsando donde el objetivo general es la atención personal, con un enfoque familiar y comunitario incluyendo el medio ambiente (MINSA-GRUN, 2007)

\section{METODOLOGÍA}

El Modelo de Persona, Familia, y Comunidad en salud ha sido impulsado desde el año 2008 en todo el territorio Nacional, con la participación de todos los trabajadores de la salud tantos del SILAIS, MINSA, como también las distintas organizaciones de la comunidad las cuales son los consejos de poder ciudadano y los gabinetes de familias. Todos estos con coordinación de parte del Gobierno Central. Este modelo se ejecuta en el área urbana y rural del territorio nacional, para posibilitar la participación de los diferentes sectores sociales de la comunidad nicaragüense.

\section{Caracterización del contexto donde se ubica el objeto de estudio (Nicaragua)}

En su primera etapa de la revolución, en 1979 producto de la situación deplorable encontrada especialmente en el estado de salud de la población, los elevados niveles de desnutrición y pobreza, se inicia un proceso de transformación económica social generado por la revolución popular sandinista, así como transformaciones de las estructuras político institucional del país, en este marco el Gobierno de Reconstrucción Nacional creó por Decreto Ley el Sistema Nacional Único de Salud (SNUS). Con el desarrollo del SNUS se incorporó la participación de la comunidad en las acciones de salud, lo que se materializó en el desarrollo de redes de voluntarios, consejos populares de salud alrededor de la red de servicios y el Consejo Nacional de Salud, fortaleciendo mecanismos locales para la solución de problemas de salud. (MINSA-GRUN, 2007)

En 2008, se inició con un nuevo modelo de persona, familia y comunidad en salud, implementado por el Gobierno de Reconciliación y Unidad Nacional (GRUN) en el marco de la restitución de derechos y participación ciudadana que este promueve. Implementando la estrategia enfocado en salud a la comunidad, donde se privilegia a cada familia nicaragüense, principalmente las más vulnerables. Asimismo, aborda a las personas en todos los ciclos de vida como un proceso continuo, con momentos interrelacionados de promoción y prevención de su salud, atención al daño y rehabilitación con un enfoque preventivo amplio y con acciones dirigidas al control del medio ambiente. (MINSA-GRUN, 2007) 


\section{Etapas de la investigación}

Investigación documental: En esta etapa se describe lo que se realizó durante el inicio de la investigación realizando diferentes actividades como: Visita al Ministerio salud (MINSA) y al SILAIS Departamental para la recopilación de datos estadísticos y sobre el enfoque del modelo.

\section{Diseño y aplicación de instrumentos: Para la} recopilación de datos, se diseñaron tres guías de encuestas semi-estructuradas dirigidas a: coordinador, técnicos y protagonista. Durante el proceso de entrevista nos explicaron de cómo los objetivos del Modelo se han cumplido de gran manera, siendo los resultados una forma de demostrar cómo han influido en el mejoramiento de la salud de la población.

\section{Análisis y elaboración del informe final: El} documento final se elaboró con la información recolectada y analizada atreves de las entrevistas y la información brindada por medio de estas instituciones, cumpliéndose los objetivos planteados de la investigación y concluir con este documento investigativo.

\section{DESARROLLO}

\section{Programa Cristiano, socialista, solidario y el Plan} Nacional de Desarrollo Humano (PNDH)

El 10 de Enero del 2007 no sólo se cambió de gobierno, se inició también un cambio de valores, actitudes, prioridades, estilo de gobernar, relaciones de poder y políticas. En este proceso se está transformando el modelo de sociedad neoliberal por el Modelo Cristiano, Socialista y Solidario del Poder Ciudadano, el cual se basa en valores cristianos, ideales socialistas y prácticas solidarias que guían la construcción de círculos virtuosos de desarrollo humano con la recuperación de valores, la restitución de derechos y el fortalecimiento de capacidades que han permitido ir superando los círculos vicios de pobreza y subdesarrollo. Esto fue posible por el poder y la participación del pueblo organizado, y como resultado de esa recuperación de valores, apoyando los programas sociales y de capitalización de las familias productoras que han logrado fortalecer sus capacidades y aumentar su participación social y económica. (GRUN, 2008)

Los valores cristianos, incluyen la valoración de la vida humana como sagrada, la opción preferencial por los pobres y el respeto de la dignidad de todo ser humano sin distingo de raza, etnia, color de piel, religión, ideología, clase social, nivel de ingreso, preferencia sexual y ninguna otra forma de discriminación o explotación. El modelo se basa en el ideal socialista que brinda oportunidades de realización y producción a todas y todos, construyendo una sociedad más igualitaria mediante la redistribución del ingreso y la riqueza, a partir del apoyo a los pobres y la micro, pequeña y mediana producción, con programas gubernamentales que transforman de manera estructural la realidad de las y los nicaragüenses, con educación y salud gratuita y universal, capitalización de los pobres y subsidios para atender las necesidades básicas de las familias, sobre todo su seguridad alimentaria y nutricional.

El modelo también es socialista al construir el Poder Ciudadano como expresión de organización y movilización del pueblo con plena participación y decisión permanente en la construcción de la democracia directa. El modelo es solidario porque las políticas públicas y prácticas solidarias parten de la realidad de las familias nicaragüenses, sus necesidades, sus aspiraciones y su situación actual, así como el impacto de los procesos socioeconómicos y políticas públicas actuales y potenciales sobre las familias nicaragüenses, especialmente las más pobres. Esta solidaridad estructural se combina con solidaridad para toda familia víctima de desastres naturales, así como para todas las personas en situación de desamparo. También se manifiesta en la responsabilidad social compartida de toda la sociedad para el cuido de la 
infraestructura económica y social del país, así como para todos los bienes públicos comunes. El Modelo Cristiano, Socialista y Solidario tiene como objetivo las transformaciones estructurales para superar la exclusión y liberar el potencial de desarrollo humano de los excluidos históricamente, como los pobres, las mujeres, los jóvenes, los pueblos originarios, los afro descendientes, los discapacitados, entre otros (GRUN, 2008).

El modelo tiene un supuesto de que la única manera de transformar Nicaragua es transformando a las y los nicaragüenses, y los logros 2007-2011 lo demuestran, con más de 500,000 personas en alfabetización y post alfabetización, con la restitución del derecho humano y constitucional a la salud y educación gratuita y universal con más de 100 mil mujeres campesinas capitalizadas en el campo de Hambre Cero y más de 123 mil mujeres pequeñas empresarias capitalizadas en la ciudad con el Programa Usura Cero, entre otros logros, son ejemplos de cómo el modelo llevó a la recuperación de valores, a la restitución de derechos y el fortalecimiento de capacidades de las y los nicaragüenses. Por eso el Plan Nacional es de Desarrollo Humano y no de desarrollo económico y social.

El Modelo Cristiano, Socialista y Solidario es un nuevo paradigma, una nueva forma de entender, pensar y hacer las cosas en todos los órdenes: Político, económico, social, ecológico, entre otros; exige una relación estrecha entre valor ético y razonamiento económico, lo que ha brindado como resultado el cumplimiento en el compromiso adquirido de erradicar la pobreza. También tiene un objetivo ético que consiste en una estrategia, una visión y práctica vinculada a que la producción debe satisfacer las necesidades espirituales y materiales de todos los ciudadanos nicaragüense, priorizando la igualdad, la redistribución equitativa, el fomento de la oportunidades y las capacidades de forma justa, y el uso racional de los recursos naturales y la producción de bienes y servicios esenciales (GRUN, 2008).

\section{Plan Nacional de Desarrollo Humano (PNDH)}

Durante los 16 años de gobiernos neoliberales el pueblo de Nicaragua no sólo perdió gran parte de las conquistas económicas sociales logradas con el triunfo de la Revolución Popular Sandinista en Julio de 1979, sino que también perdió las conquistas económicas y sociales heredadas de la Revolución Liberal de 1893. Privatización del sector transporte y desaparición del ferrocarril, así como la privatización de las telecomunicaciones, energía, educación, salud y el servicio del seguro social, con resultados desastrosos, sobre todo en energía, donde no hubo inversión, lo que llevó a una crisis energética. La desaparición de la Reforma Agraria lo que aumentó la pobreza de la gran mayoría de la población. Políticas dirigidas a destruir la producción agropecuaria dejándolo sin financiamiento ni programas estructurales que promoverían su desarrollo y llamando al abandono del campo y al fortalecimiento del comercio para la importación de alimentos. (GRUN, 2008)

Todo esto es el resultado global de los 16 años de gobiernos neoliberales lo cual conllevo al empobrecimiento del país, colocándolo como el segundo país más pobre de América Latina y el Caribe, solo Haití tiene mayor nivel de pobreza. Es por eso que el GRUN se vio a la necesidad de realizar un cambio de Modelo. Después de 16 años de aplicación del Modelo Neoliberal, el Gobierno de Reconciliación y Unidad Nacional comenzó a construir desde el primer día en el gobierno el Modelo de Desarrollo del Poder Ciudadano, un modelo integraly humanista para superar la pobreza en el segundo país más pobre de América Latina. El punto de salida y llegada de este modelo es el ser humano nicaragüense en las condiciones históricas, culturales, sociales y políticas nacionales y regionales. El nuevo Modelo de Desarrollo del Poder Ciudadano valora que la vida humana es sagrada y en consecuencia tiene a la persona, hombres y mujeres como el centro y el sujeto fundamental del Desarrollo, a fin de que se pueda disfrutar de una vida prolongada y 
saludable, adquirir conocimientos y tener acceso a los recursos necesarios para lograr un nivel de vida digno que permita su plena realización según sus sueños.

En el marco de estos valores y prioridades, el nuevo modelo tiene como objetivo transformar el modelo neoliberal, a un nuevo modelo cuya finalidad y prioridad es la supervivencia y la realización de los seres humanos, así como la superación de la pobreza. Creando transformaciones estructurales para superar la exclusión y liberar el potencial de desarrollo humano de los excluidos, incluyendo los pobres, las mujeres, los jóvenes, los pueblos originarios, los afro descendientes, los discapacitados, entre otros., creando condiciones para la plena realización de todos los nicaragüenses en un país soberano e independiente en unión con los hermanos centroamericanos, caribeños y latinoamericanos (GRUN, 2008).

\section{Caracterización Modelo de persona, familia y comunidad en Salud.}

\section{Antecedentes}

A inicios del Siglo XX, la atención en salud se había caracterizado por estar fragmentado, centralizado y desarticulado en la provisión de servicios de salud, que limitaban la posibilidad de una atención integral a la población. En este sentido, las políticas anteriores a este proceso, de corte neoliberal fueron muy ineficaces lo cual con conllevó a profundizar la inequidad en el acceso a los servicios de salud de los grupos poblacionales en situación de pobreza y extrema pobreza. Una visión global de estos países (Guatemala, Perú y Nicaragua) muestra que en las tres últimas décadas sus políticas de salud han tenido un gran realce, que logran un impacto real y positivo en el cumplimiento del derecho a la salud de sus respectivas poblaciones.

En el Perú, desde hace varios años, existieron esfuerzos importantes por introducir la atención integral en los servicios. La mayoría de las experiencias se iniciaron en la primera mitad de la década de los 90, habiendo alcanzado su mayor desarrollo en los últimos años de ésta. El Programa de Salud Básica para Todos (PSBPT) desarrolló una metodología para la formación de recursos humanos orientados a prestar una atención integral. En un primer momento, se concibió la atención integral como la prestación integrada de los servicios que eran ofrecidos por los programas nacionales entonces vigentes. Se buscaba generar mayor eficiencia y reducir las oportunidades perdidas que iban contra el cumplimiento de las metas programáticas. Estos esfuerzos incluyeron la definición de estándares y mecanismos para la acreditación de servicios que cumplían con los criterios de atención integral. (Caro, 2010)

En el caso de Guatemala, en 1,996 dentro del marco de la Reforma del Sector Salud y los Acuerdos de Paz, nace el Programa de Extensión de Cobertura (PEC), para mejorar el acceso de los servicios del Primer Nivel de Atención dirigido a las poblaciones rurales y postergadas, que contribuyen a reducir la mortalidad materna infantil y los problemas de salud prioritarios. Se desarrollan programas de promoción de la salud, prevención primaria y saneamiento básico. (MSPAS, 2016)

Durante los años 2005, 2006 y 2007, se continuó trabajando en el diseño e implementación de un Modelo Básico de Gestión y Atención Integral en Salud, que estructuraba la organización del nivel operativo, representado por las Áreas de Salud en cuatro Gerencias, consistentes en Provisión de Servicios, Control y Vigilancia de la Salud, de Recursos Humanos y Administrativo Financiera, planteándose los recursos pertinentes para el fortalecimiento de su capacidad operativa y se integraron los equipos de seguimiento, pero por diversas razones no se consolidó. Además, en el ámbito del Modelo de Atención, se organizó la red de servicios en tres Niveles de Atención y se actualizaron las normas programáticas para ser 
implementadas en Centros y Puestos de Salud, con la respectiva capacitación al personal mediante un Diplomado, proceso que tampoco tuvo el seguimiento necesario para verificar su cumplimiento y apropiación. (MSPAS, 2016)

En Nicaragua, la atención en salud era garantizada por médicos de cabecera en las principales áreas urbanas. Los hospitales eran centros de atención a indigentes, regentados por órdenes religiosas y con el apoyo filantrópico de personas pudientes de la sociedad y la colaboración profesional desinteresada de los médicos locales. Las acciones de salud pública, fundamentalmente dirigidas a la higiene comunal eran regidas por el reglamento de policía publicado por decreto del 25 de octubre de 1880 y sus modificaciones de mayo de 1938. Grandes grupos de población eran atendidos por agentes tradicionales como parteras, curanderos y brujos. (MINSA-GRUN, 2007)

En 1979, producto de la situación deplorable encontrada especialmente en el estado de salud de la población, los elevados niveles de desnutrición y pobreza, se inicia un proceso de transformación económica social generado por la revolución popular sandinista, así como transformaciones de las estructuras político institucional del país, en este marco el Gobierno de Reconstrucción Nacional creó por Decreto Ley el Sistema Nacional Único de Salud -SNUS (Decreto Presidencial No 35 del 8 de Agosto), que unificó bajo administración del Ministerio de Salud a todos los establecimientos prestadores de servicios de salud pública y privados, incluyendo los servicios del INSS los cuales pasaron a ser parte del SNUS. Con el desarrollo del SNUS se incorporó la participación de la comunidad en las acciones de salud, lo que se materializó en el desarrollo de redes de voluntarios, consejos populares de salud alrededor de la red de servicios y el Consejo Nacional de Salud, fortaleciendo mecanismos locales para la solución de problemas de salud. (MINSA-GRUN, 2007)
El Ministerio de Salud de esta forma se convirtió en el prestador exclusivo de servicios de salud para toda la población, impulsó un proceso de extensión de cobertura en todo el país, fundamentalmente en cuanto a servicios de primer nivel de atención a la luz de la recién adoptada meta "Salud Para Todos en el año 2000" y de la estrategia de Atención Primaria en Salud. (MINSA-GRUN, 2007)

\section{Estrategia organizativa del Modelo de persona, familia y comunidad en salud (MINSA) ${ }^{1}$}

El Modelo de Salud Familiar y Comunitario está basado en la estrategia de atención primaria en salud, entendida como "la asistencia sanitaria esencial basada en métodos y tecnologías prácticas, científicamente fundamentado y socialmente aceptada, puesta al alcance de todos las personas y familias de la comunidad, mediante su plena participación y a un costo que la comunidad y el país puedan soportar en todas las etapas de su desarrollo, con espíritu de auto responsabilidad y autodeterminación". Para mejorar la salud, la atención primaria se centra en la salud de las personas en los contextos de los otros determinantes; es decir, en el medio físico y social en el que las personas viven, estudian, trabajan y se recrean, más que en enfermedades específicas. Los principios del modelo son:

- Accesibilidad a los servicios de salud: El ordenamiento de los servicios de salud se basará en criterios de accesibilidad geográfica, cultural, de género, económica, etc. que garanticen una puerta de entrada para favorecer el primer contacto de los usuarios, incluyendo a las comunidades indígenas y grupos étnicos, a fin de fomentar la confianza y credibilidad de los servicios. El Modelo de Salud Familiar y Comunitario debe concretizar los

1 MINSA-GRUN. (2007). Marco conceptual del Modelo de Salud Familiar y Comunitaria (MOSAFC) (Vol. 2da. edición). Managua, Managua, Nicaragua. Obtenido de http://www.paho. org/nic/index.php?option=com_docman\&task=doc_view\&gi$\mathrm{d}=230$ \&Itemid $=235$ 
principios de gratuidad, universalidad, solidaridad y equidad. Asimismo, se asume el principio de accesibilidad de la Política Nacional de Población

- Integralidad de las acciones en los servicios de salud: El Modelo de Salud Familiar y Comunitario contemplará a la persona como un ser biopsicosocial perteneciente a una familia y a una comunidad, con deberes y derechos para la toma de decisiones de forma consciente y sistemática respecto a su salud, así como para la protección y mejora del ambiente que lo rodea. Implica la orientación de la atención hacia la solución integral de los problemas de salud, con enfoque de promoción, prevención de enfermedades, curación y rehabilitación de la salud. Asegura que distintos servicios lleguen al mismo tiempo y se realicen en los territorios donde las personas y/o grupos poblacionales.

- Longitudinalidad (continuidad) en el proceso de la atención en salud: Es la relación personal a largo plazo que se establece entre el personal proveedor y usuarios del servicio de salud. Implica que el equipo de salud al cuidado de los usuarios se preocupe de su atención integral en los diferentes niveles de atención. Esta característica del modelo de atención nicaragüense es uno de los pilares para asegurar la articulación entre los diferentes niveles de atención y la cohesión de la red de servicios de salud del país y, por tanto, de la referencia y contra referencia. La atención longitudinal significa que los usuarios pertenecientes a una población identifiquen como suya a una red de servicios, su establecimiento inmediato de atención y personal encargado de proveerle servicios.

- Coordinación entre los niveles de atención en salud: El sector de salud nicaragüense, al organizarse en redes de servicios por niveles de atención y con establecimientos de salud de complejidades diversas, requiere tener identificación clara de la población a atender, la cual puede acudir y acceder a los establecimientos para ser atendida en diversos aspectos de sus necesidades de salud. La coordinación debe ser lo suficientemente efectiva cómo para garantizar el acceso efectivo y la continuidad de la atención.

\section{Principales resultados del Modelo de persona, familia y comunidad en salud ${ }^{2}$}

Casa Base: Es una forma de organización comunitaria que tiene como propósito impulsar el autocuidado de la salud, implementando acciones permanentes de prevención, promoción y asistencia básica a los principales problemas de salud, a través de voluntarios de la comunidad y en coordinación con los equipos de salud familiar de los Puestos de Salud Familiar y Comunitarios.

Casa Materna: Es un establecimiento de salud comunitario que tiene como propósito acercar a la mujer que reside en zonas distantes a servicios de salud con complejidad adecuada para atender con personal calificado el embarazo, parto, recién nacido y puerperio de bajo riesgo, para su debida atención segura con calidad y respeto a su condición socioeconómica, cultural, étnica y otras características de estas mujeres y sus familias.

Como resultados utilizando estas dos estrategias (Casa Base y Casa Materna) se han disminuido considerablemente la mortalidad infantil en Nicaragua, en años anteriores (1990-2001) se tenía una mortalidad infantil del $67 \%$ al $31 \%$, actualmente se tiene un $19 \%$ de la mortalidad infantil a nivel Nacional. Como otros resultados se ve la atención que han beneficiado a las mujeres embarazadas, ya que se ha incrementado las mujeres que han sido albergadas en estas distintas casas maternas, en el 2006 se tenía albergado anualmente a 9,205 mujeres para el 2015 se habían albergado a más de 51,189 mujeres, esto demuestra la calidad del servicio de salud que se les brinda estas madres. Por lo

2 MINSA-GRUN. (2007). Marco conceptual del Modelo de Salud Familiar y Comunitaria (MOSAFC) (Vol. 2da. edición). Managua, Managua, Nicaragua. Obtenido de http://www.paho. org/nic/index.php?option=com_docman\&task=doc_view\&gi$\mathrm{d}=230$ \&Itemid $=235$ 
tanto, la supervivencia infantil incremento en los años 1992-1993 de cada 1,000 niño sobrevivía 942, en el año 2015 de cada 1,000 sobrevivía 983 niños.

Puesto de Salud Familiar y Comunitario: Es la unidad funcional básica dentro de la red municipal del Ministerio de Salud está ubicado en áreas rurales o urbanas. $\mathrm{Su}$ principal objetivo es responder con la participación comunitaria al reto de preservar la salud y enfrentar los principales problemas de salud de la población. Su actividad está orientada a la promoción, prevención y atención oportuna al daño. Atiende emergencias relativas, efectúa referencias y da seguimiento a las contra referencias de pacientes.

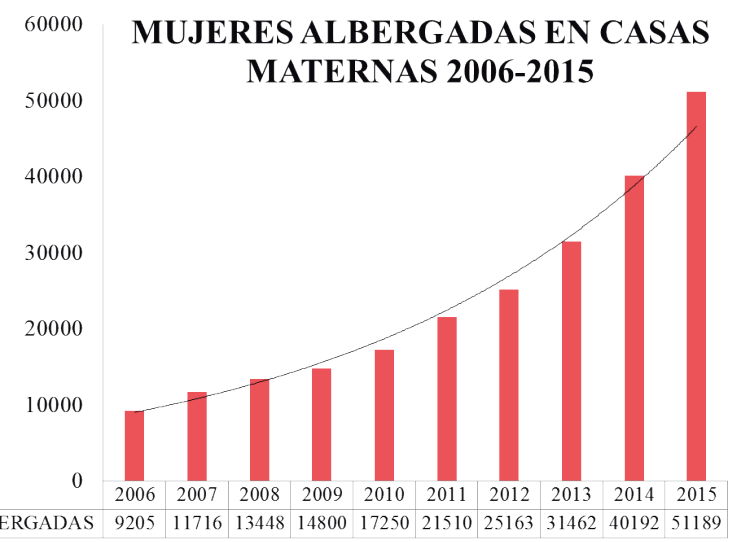

Fuente: MINSA, 2016

Centro de Salud Familiar: Es una unidad de salud de atención ambulatoria que proporciona servicios orientados a la promoción, prevención y atención oportuna al daño, según lo definido en el conjunto de prestación de servicios y acorde a la demanda. Es la unidad de provisión y gestión de los servicios ambulatorios de atención integral a las personas, familia y comunidad; así como a la salud ambiental que jerarquiza la red de servicios de su Municipio o Distrito.

Hospital Primario: Son unidades de salud que además de cumplir las funciones de un Centro de Salud Familiar, brindan servicios de internamiento en pediatría y ginecoobstetricia, con un servicio de observación para adultos en medicina y cirugía general. Se encuentran ubicados en cabeceras municipales con áreas de influencia eminentemente rurales, distintas a la cabecera Departamental. Para cumplir sus funciones pueden tener entre 15 y 40 camas como máximo para internamiento dependiendo de la población de los municipios de su área de influencia y cumple con los estándares de habilitación establecidos.

Como mayor resultado de la creación de estos nuevos centros de salud se ha incrementado los trabajadores del servicio de la salud, en 2006 se contaba con 22,083 personal de salud, en 2015 incremento al 32, 289 aumentando un $42.2 \%$ en personal de salud. Otro sector que se vio reflejado fue en el aumento de médicos en el MINSA, para el año 1977 se constaba con 1,319 en 2015 se cuenta con 5,794 médicos, en el caso del área de enfermería también ha venido incrementando para el año 1977 habían 3,505, en 2015 se contaba con 8,279 . Queda entrevisto que a la comunidad le gusta cómo se trabaja con este nuevo modelo.

No solo se notan los resultados en el área de los trabajadores de la salud, sino también en el acceso de la población a los hospitales públicos, por ejemplo los partos atendidos en los hospitales públicos con relación al años 1998 donde el 55.8\% era atendidas por parteras, en el 2015 son atendidas por parteras el 20.7\% es decir que hubo una disminución del $53 \%$, el cual este porcentaje de la población está siendo atendido en los hospitales públicos. Otros avances que se ha tenido con este modelo, es la capacitación precoz con la atención prenatal, con relación al 2006 el 39\% de las mujeres embarazadas ya iban preparadas, actualmente el $55 \%$ ya tiene conocimiento alguno. Los controles de las mujeres han cambiado, antes se realizaban más de 4 controles en su periodo de embarazo, actualmente se elaboran 4 controles pero multidisciplinarios donde todo un equipo conformado por enfermeras y médicos hacen una revisión general del estado de salud del bebe y de la madre 


\section{Número de Médicos del MINSA}

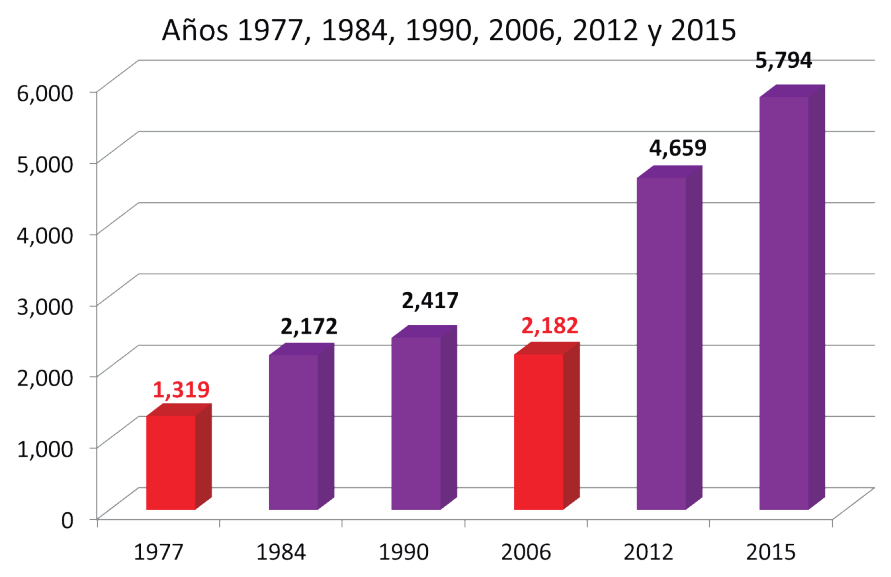

Fuente: MINSA, 2016

\section{Participación ciudadana en el Modelo de persona, familia y comunidad en salud (MINSA)}

Dado el rol relevante de la participación ciudadana en el proceso de transformación del sistema de salud y su posicionamiento en la estructura territorial, es importante considerar lo establecido en la Ley 475 de participación ciudadana, en la que se define como un proceso de involucramiento de actores sociales en forma individual o colectiva, con el objeto y finalidad de incidir y participar en la toma de decisiones, gestión y diseño de las políticas públicas en los diferentes niveles y modalidades de la administración del territorio nacional y las instituciones públicas, con el propósito de lograr un desarrollo humano sostenible en corresponsabilidad con el Estado. Para ejercer con responsabilidad la participación ciudadana en el desarrollo local existen diferentes espacios de concertación constituidos en los diferentes niveles territoriales, como los consejos comunitarios, redes territoriales, Consejos del Poder Ciudadano (CPC), gabinetes comarcales, mesas de concertación nacional, departamental, regional o municipal, que permiten la planificación, co-ejecución de acciones y el desarrollo del control social. También se crean dentro del sistema de salud estos mecanismos de coordinación de la gestión en salud.
El Equipo de Salud Familiar brinda un conjunto de prestaciones de servicios de salud dirigidas a las Personas, Familias y Comunidad. La finalidad es la promoción y prevención de la salud y el tratamiento adecuado que incluye la recuperación y rehabilitación de la población asignada a los sectores, involucrando en su realización la actuación de la comunidad organizada, los diversos actores sociales comprometidos en el mejoramiento de la calidad de vida de la población, de acuerdo a su realidad y necesidad.

- Comités Comunitarios: Garantizan la organización de la comunidad motivando su participación activa en el desarrollo para lograr una vida mejor. Fortaleciendo la organización y funcionamiento permanente del comité con participación de todos sus miembros, a través de su capacitación para mejorar actitudes y comportamientos en las familias y miembros de las comunidades Promoviendo la elaboración de los planes comunitarios, su seguimiento y evaluación efectiva en coordinación con todos los actores, dando a cada quien lo que necesita.

- Intersectorial: Se lograráa través de coordinaciones y alianzas estratégicas que los diversos sectores establecen dentro de su ámbito de competencia, alrededor del cumplimiento de objetivos, implementación y evaluación de políticas, planes y programas de salud que se desarrollan bajo la rectoría y el liderazgo del MINSA. Con este fin se aprovechará las estructuras existentes, como los Consejos Departamentales y Municipales de Salud.

- Interinstitucional: Se desarrolla a través de coordinaciones y alianzas estratégicas, teniendo como punto de partida la identificación de objetivos comunes para la realización de acciones de promoción y prevención. La participación interinstitucional procurará superar o reducir la brecha en la cobertura de programas y servicios básicos, mejorar la efectividad y calidad técnica percibida de los servicios de salud. 
Para garantizar la sostenibilidad y el desarrollo del Modelo de Salud Familiar y Comunitario se hace necesario impulsar procesos de dirección colectiva en los que la amplia participación de directores de todas las unidades de gestión del SILAIS aporte en la valoración de los aciertos, limitaciones y problemas para el cumplimiento de los objetivos y metas definidas. Esta dirección colectiva se garantiza en los Consejos técnicos en los cuales se debate acerca de la situación en el SILAIS y en forma colegiada se decidan las intervenciones a implementar, con el compromiso de todos por el éxito de las mismas. Las herramientas para ejercer el control de gestión son las siguientes: Sistema de Información en Salud y Sistema de Garantía de la Calidad Procesos evaluativos.

\section{CONCLUSIONES}

El modelo de Persona, Familia y Comunidad (MINSA) en Nicaragua es un ejemplo de cambio y sobre todo de respuestas, ante la precariedad del sistema de Salud durante los gobiernos Neoliberales, el Gobierno de Reconciliación y Unidad Nacional fomenta la participación de la ciudadanía como eje de desarrollo para el éxito de este modelo familiar. El Modelo de Salud Familiar y Comunitario contemplará a la persona como un ser biopsicosocial perteneciente a una familia y a una comunidad, con deberes y derechos para la toma de decisiones de forma consciente $\mathrm{y}$ sistemática respecto a su salud, así como para la protección y mejora del ambiente que lo rodea. Implica la orientación de la atención hacia la solución integral de los problemas de salud, con enfoque de promoción, prevención de enfermedades, curación y rehabilitación de la salud. Asegura que distintos servicios lleguen al mismo tiempo y se realicen en los territorios donde las personas y/o grupos poblacionales lo necesiten. Esto implica responsabilidad territorial y poblacional por parte de los diferentes actores claves del sector social.

Dado el rol relevante de la participación ciudadana en el proceso de transformación del sistema de salud y su posicionamiento en la estructura territorial, es importante considerar lo establecido en la Ley 475 de participación ciudadana, en la que se define como un proceso de involucramiento de actores sociales en forma individual o colectiva, con el objeto y finalidad de incidir y participar en la toma de decisiones, gestión y diseño de las políticas públicas en los diferentes niveles y modalidades de la administración del territorio nacional y las instituciones públicas, con el propósito de lograr un desarrollo humano sostenible en corresponsabilidad con el Estado.

Para ejercer con responsabilidad la participación ciudadana en el desarrollo local existen diferentes espacios de concertación constituidos en los diferentes niveles territoriales, como los consejos comunitarios, redes territoriales, Consejos del Poder Ciudadano (CPC), gabinetes comarcales, mesas de concertación nacional, departamental, regional o municipal, que permiten la planificación, co-ejecución de acciones y el desarrollo del control social. También se crean dentro del sistema de salud estos mecanismos de coordinación de la gestión en salud. Es por eso que el modelo alcanzado un gran realce en Nicaragua, porque se ve como los distintos sectores sociales de Nicaragua participa de la mano con el ministerio de salud y con el gobierno central para el mejoramiento de la comunidad.

\section{BIBLIOGRAFÍA}

MSPAS. (2016). Modelo de atención del primer nivel (Primera edición ed.). (M. d. Social, Ed.) Guatemala, Guatemala. Obtenido de http://www. mspas.gob.gt/files/Descargas/UNIDADES/ primernivel/MODELOATENCION1ERNivel\%20 final.pdf

Caro, R. E. (2010). Modelo de atención integral de salud en la percepción de los directivos de la Dirección Regional, Hospitales y Centros de Salud del Ministerio de Salud del Departamento del ICA. 2007. Lima, Perú. Obtenido de http://cybertesis. 
unmsm.edu.pe/bitstream/cybertesis/1334/1/Caro rr.pdf

CEPAL. (2013). Sistema de las Naciones Unidas en Chile. (CEPAL) Obtenido de http://www.onu.cl/ onu/sample-page/agencias-fondos-y-programas/ cepal/

GRUN. (2008). Plan Nacional de Desarrollo Humano 2008-2012. Managua Nicaragua: Gobierno Nacional. Obtenido de http:// www.pndh.gob.ni/documentos/Plan \% 20 Nacional\%20de\%20Desarrollo\%20Humano\%20 2008-2012-Nicaragua.pdf

Gudynas, E. (2009). La CEPAL, ¿realmente entiende los problemas actuales del desarrollo en América Latina? Obtenido de http://www.rebelion.org/ noticia.php?id=97212
Martner, R., \& Mattar, J. (2012). Los fundamentos de la planificación del desarrollo en América Latina y El Caribe. Textos seleccionados ILPES (1962-1972). Santiago de Chile, Chile: Libros de la CEPAL. Naciones Unidas. Obtenido de http:// archivo.cepal.org/pdfs/2012/S2012845.pdf

MINSA-GRUN. (2007). Marco conceptual del Modelo de Salud Familiar y Comunitaria (MOSAFC) (Vol. 2da. edición). Managua, Nicaragua. Obtenido de http://www.paho.org/nic/index.php?option=com docman\&task $=$ doc_view\&gid=230\&Itemid $=235$

Sanchez, E. (2009). Papel de la CEPAL y el FMI en el desarrollo económico de América Latina 2005 a 2008. Obtenido de http://papel-de-lacepaly-el-fmi-en-al.blogspot.com/2009/05/trabajocolaborativo-de-macroeconomia.html 